potentially useful as screening tests for early-stage dementia, these methods remain less utilized in Asian countries due to limited resources.

\section{S3-01-04 CULTURAL EFFECT OF ALZHEIMER'S DISEASE IN THE NATIVE AMERICAN POPULATION}

\section{J. Neil Henderson, L. Carson Henderson; University of Oklahoma Health Sciences Center, Oklahoma City, OK, USA}

Background: Multifactorial etiologic pathways leading to dementing diseases provoke the need to expand research horizons beyond conventional studies in majority population subjects. The bulk of dementia research in the U.S. has been done with majority population subjects. Corrective action requires that research in understudied populations must be accelerated. This is particularly true with indigenous people of the U.S. because of unique genetic loading relative to dementia, high prevalence of cardiovascular disease associated with dementia, and pluralistic health belief systems. Objective(s): Ascertainment of APO-E and tau allele frequency as a function of genetic loading of indigenous genes to non-indigenous genes (i.e., blood quantum), description of explanatory models of dementia, and development of a multifactorial model of dementia from these data derived from a large Federally recognized tribe. Methods: Subject recruitment was purposive (certified tribal membership, over age 21, tribal health care staff assisted in subject identification) and gained 172 subjects. Genotyping was done from blood samples. Cognitive status was screened using the "Screening for Memory Disorder Protocol" (MMSE, geriatric depression scale and the Short Portable Mental Status Questionnaire). Explanatory models were collected by personal interview done by an anthropologist who is also a member of this tribe. Conclusions: Compared to whites, APO-E4 and tau alleles are about one-half as frequent (6\%), subjects with less than .5 genetic loading of indigenous genes have the same association of between the APO-E4 genotype and disease ( $\mathrm{P}<0.05)$, APO-E4 appears to be less potent in contributing to $\mathrm{AD}$ in either homozygous or heterozygous conditions, and these factors vary inversely by degree of indigenous ancestry. Explanatory model investigation produced local indigenous cultural beliefs characterizing a range of attributional models, including a unique nonpathological schema. Additionally, a multifactorial, biocultural hypothesis for the etiology of dementia shows a chain of genetic, morbidity and behavioral factors each of which is postulated to contribute additively to dementia. The behavioral factors associated with management of diseases that increase risk for dementia constitute potential target areas for reducing the risk of late life dementia.

\section{TUESDAY, JUNE 21, 2005 SYMPOSIA \\ S3-02 TREATMENTS IN THE FUTURE}

\section{S3-02-01 THE POTENTIAL ECONOMIC IMPACT OF FUTURE TREATMENTS FOR DEMENTIA}

Kenneth M. Langa; University of Michigan, Ann Arbor, MI, USA

Background: Economic analyses of treatments for dementia are complex, and often subject to conflicting interpretations, due to unique aspects of the epidemiology and clinical course of cognitive decline, as well as a number of methodological issues. Objective(s): This presentation will review health economic issues in the prevention and treatment of dementia, including challenges in: 1) measuring quality of life for patients and caregivers; 2) identifying clinically significant effects of treatment; 3) estimating the time and value of informal caregiving; and 4) accounting for multiple co-existing conditions in older individuals at-risk for dementia. Methods: A review of health economic concepts, as well as a focused literature review of recent studies of the cost-effectiveness of treatments for dementia. Conclusions: Dementia has a profound economic impact on patients, families, and social programs. Measuring the economic impact of current and future treatments for dementia requires careful attention to the unique aspects of dementia epidemiology and clinical course.

\section{S3-02-02 EARLY DETECTION OF COGNITIVE DECLINE WITH EMBEDDED ASSESSMENT}

Margaret E. Morris; Proactive Health, Intel Research, Hillsboro, OR, USA

Background: Early detection of cognitive decline is critical if emerging medical treatments are to effectively treat individuals who are vulnerable to Alzheimers disease and other forms of dementia. Current assessment practices typically permit diagnosis only after the significant disease progression. By continuously monitoring behavior and cognition in the home environment, pervasive computing may help identify people at risk for decline. Such systems need to accommodate attitudes about health monitoring and early detection, as well as individuals' immediate and long-term concerns regarding health, performance, and quality of life. Objective: To describe a model and examples of cognitive assessment that is embedded in individuals' home environments as well as their preventative and compensatory strategies. Methods: The embedded assessment approach evolved from ethnographic home interviews with cognitively impaired patients, spouse-caregivers, and healthy adults. These interviews focused on behaviors and attitudes preceding and following a diagnosis of cognitive decline. In subsequent phases of research, healthy and cognitively impaired participants responded to descriptions, sketches and live enactments of embedded assessment concepts. In-home case studies with 6 elder-caregiver dyads were used to test one early stage prototype. Conclusions: There is a need for household cognitive assessment embedded within preventive strategies and supportive aids. An embedded approach will resolve the tension between individuals' avoidance of diagnosis and their desire for prognostic, compensatory and preventative guidance. Computing technologies in the home may determine subclinical changes based on personalized baselines of functioning while providing assistance in daily life and ideally also helping people maintain cognitive health. Embedded assessment should support non-medical domains, such as social connectedness and fitness, which have preventative value throughout the lifespan.

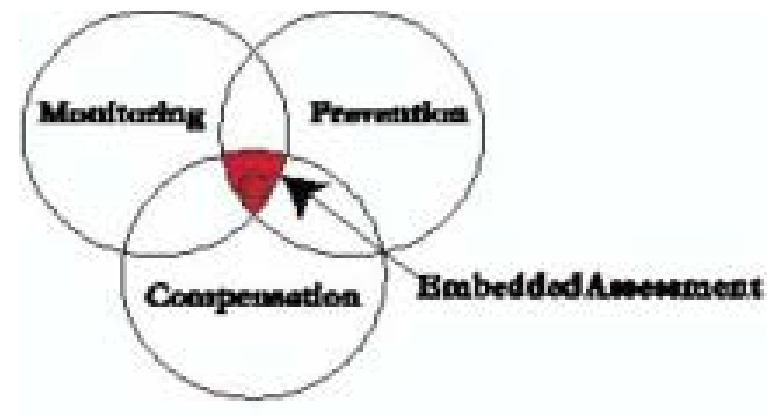

S3-02-03 AD VACCINATION USING HSV AMPLICONS

Howard Federoff; Neurology Center Aging \& Development, Rochester, NY, USA

Background: Vaccination methods for Alzheimer's disease have frequently relied upon peptide and adjuvant approaches. This method has elicited both humoral and cellular immune responses in animal models and human subjects. Herein we employ a novel vaccine based upon helper virus free HSV amplicons. Objective(s): We sought to determine whether HSV Amplicons harboring $\mathrm{A} \beta$ or $\mathrm{A} \beta$ fused to the molecular adjuvant tetnus toxin $C$ light chain fragment (TTC) would provide protective immune responses in the $\operatorname{Tg} 2576$ and other AD models. Methods: $\mathrm{Tg} 2576$ mice were vaccinated thrice with HSV amplicons of each type. Immune responses and $\mathrm{A} \beta$ burden were determined. Conclusions: Vaccination with HSVA $\beta$ alone produced CNS inflammation, mortality and an apparent Th1 